\title{
Abl Tyrosine Kinase Promotes Dendrogenesis by Inducing Actin Cytoskeletal Rearrangements in Cooperation with Rho Family Small GTPases in Hippocampal Neurons
}

\author{
Shiloh B. Jones, Hope Y. Lu, and Qun Lu \\ Department of Anatomy and Cell Biology, The Brody School of Medicine at East Carolina University, Greenville, North Carolina 27858
}

\begin{abstract}
Nonreceptor tyrosine kinase $\mathrm{Abl}$ is an actin-binding protein and a key regulator of neuronal axonal development. Although Abl family kinases also are localized in dendrites and are implicated in postsynaptic functions, it is not clear how Abl kinases regulate dendritic morphogenesis. Using a developing hippocampal culture as a model, we found that the inhibition of Abl kinases by STI571 leads to a remarkable simplification of dendritic branching similar to the phenotype caused by an increased activity of small GTPase RhoA. Time-lapse microscopic imaging reveals a prominent reduction of dendritic branching. In contrast, neurons expressing a constitutively active v-abl construct (CA-Abl) show an exuberant microtubule-associated protein 2-positive (MAP2-positive) dendrite outgrowth, suggesting that $\mathrm{Abl}$ modulates dendritic growth. Biochemical assays using a glutathione $S$-transferase pull-down method to determine GTP-bound active Rho GTPases demonstrate that Abl inhibition increases RhoA activity but has no effect on the activity of Rac1 or Cdc42. At the cellular level the alteration of Abl also changes actin organization consistent with RhoA inhibition. Suppression of the RhoA downstream effector Rho kinase reverses STI571-induced dendritic simplification, demonstrating that activity of the Rho pathway is responsible for the Abl-induced changes in dendrogenesis. Furthermore, CA-Abl-induced neurite outgrowth is blocked by the expression of a constitutively active RhoA construct. The CA-Abl phenotype is not affected by destabilization of microtubules but is reversed partially when actin filaments are stabilized with jasplakinolide. Together, these studies support a critical role for Abl kinases in regulating dendrogenesis by inducing actin cytoskeletal rearrangements in cooperation with Rho GTPases.
\end{abstract}

Key words: nonreceptor tyrosine kinase; neuronal morphogenesis; Rho/Rac/Cdc42; actin cytoskeleton; Rho kinase; STI571/Gleevec/ imatinib mesylate

\section{Introduction}

The development of functional circuitry in the CNS is an intricate process that relies, in part, on the morphological complexity of resident neurons. Dendrites act as integrators of synaptic input; thus the process of dendrogenesis determines the number and pattern of synapses that are received by each mature neuron (McAllister, 2000). Development of the dendritic tree is a series of dynamic events that result in the formation of a complex and highly ordered structure through abundant remodeling and reorganization of the actin and microtubule cytoskeletons. These processes are regulated by a variety of extracellular signals, which eventually converge on signaling pathways that effect cytoskeletal reorganization (Luo, 2000; Rao and Craig, 2000; Pendergast, 2002).

The Abl family of nonreceptor tyrosine kinases is an important link in signal transduction pathways that promote cytoskel-

Received April 3, 2004; revised July 2, 2004; accepted July 28, 2004.

This study was supported in part by The American Cancer Society and The Brody School of Medicine Research Award (to Q.L.). We thank Drs. D. Baltimore, K. Burridge, and D. Kufe for providing cDNA constructs; J. Griffin, R. Nowakowski, and Y. H. Chen for suggestions; Novartis for providing STI571; and members of the Lu laboratory for technical assistance and discussions.

Correspondence should be addressed to Dr. Qun Lu, Department of Anatomy and Cell Biology, Brody School of Medicine at East Carolina University, Greenville, NC 27858. E-mail: luq@mail.ecu.edu.

DOI:10.1523/JNEUROSCI.1264-04.2004

Copyright $\odot 2004$ Society for Neuroscience $\quad 0270-6474 / 04 / 248510-12 \$ 15.00 / 0$ etal rearrangement (Lanier and Gertler, 2000; Pendergast, 2002). During brain development Abl family kinases play an important role in neurulation, because abl/arg double-null mice exhibit defective neural tube morphology and gross alterations in neuroepithelial actin cytoskeleton structures (Koleske et al., 1998). Abl kinases are involved additionally in moderating actin dynamics in axonogenesis and growth cone motility, and they have been shown to stimulate neurite outgrowth in primary neuronal cultures (Zukerberg et al., 2000; Woodring et al., 2002). Abl localization also has been demonstrated in the growth cones of primary hippocampal neurons where it interacts with $\delta$-catenin, a dendrite-specific Abl substrate that recently has been shown to affect dendrite outgrowth and branching (Kim et al., 2002; Lu et al., 2002; Martinez et al., 2003). In addition, Abl kinases are localized to both presynaptic and postsynaptic sites in mature neurons and at the neuromuscular junction (Finn et al., 2003; Moresco et al., 2003). A precise function for Abl in developing dendrites, however, is undefined.

Abl kinase regulates actin-dependent processes via interactions with several protein families, including the Rho family small GTPases (Van Etten, 1999; Lanier and Gertler, 2000; Liebl et al., 2000). For example, Abl kinases may decrease RhoA activation via the phosphorylation of an upstream Rho regulator, p190Rho GTPase-activating protein (p190RhoGAP) (Moresco and Koleske, 
2003). Signaling pathways involving the Rho family of small GTPases mediate distinct actin cytoskeleton reorganization events in different cell types and have been proposed to be key mediators of dendritic development. In particular, expression of constitutively active RhoA inhibits dendrite growth and induces neurite retraction (Katoh et al., 1998; Lee et al., 2000; Luo, 2000; Nakayama et al., 2000). Conversely, RhoA null mutations promote dendrite outgrowth and cause overextension of dendritic processes (Lee et al., 2000). Active RhoA can initiate cellular process retraction by acting on Rho-associated kinase/p160/ROCK (Katoh et al., 1998), which can be prevented by inhibition of ROCK activity (Bito et al., 2000; Da Silva et al., 2003). Therefore, it is possible that Abl may cooperate with signaling partners of Rho family small GTPases to contribute to dendrogenesis. In this study we demonstrate that Abl family nonreceptor tyrosine kinases promote dendrogenesis via a RhoA/ROCK-dependent manner in developing hippocampal neurons.

\section{Materials and Methods}

Hippocampal cultures, transfections, and pharmacological treatments. Embryonic hippocampal cultures were prepared as described by Goslin and colleagues with minor modifications (Goslin et al., 1998; Jones et al., 2002; Lu et al., 2002). Briefly, $18 \mathrm{~d}$ timed pregnant rats were killed, and the embryos were removed in accordance with the National Institutes of Health Guide for the Care and Use of Laboratory Animals. Hippocampi were collected, and cells were dissociated by trypsinization and plated onto poly-L-lysine-coated coverslips in DMEM supplemented with $10 \%$ FBS. After neurons adhered to the substrate, the medium was changed to Neurobasal supplemented with B-27. This culture scheme allowed us to maintain viable low-density hippocampal cultures for several weeks.

Hippocampal neurons grown for $5 \mathrm{~d}$ in vitro (5 DIV) were transfected with enhanced green fluorescent protein (pEGFP; Clontech, Palo Alto, CA), using Lipofectamine 2000 reagent (Invitrogen, Grand Island, NY), according to the instructions provided. Constitutively active v- $a b l$ and constitutively active RhoA-GFP (Q36L) kindly were provided by D. Baltimore and $\mathrm{K}$. Burridge, respectively. The neurons were fixed in $4 \%$ paraformaldehyde $48 \mathrm{hr}$ after transfection. Alternatively, neurons were subject to $100 \mathrm{~nm}$ jasplakinolide (Molecular Probes, Eugene, OR) for 5 hr, $5 \mu \mathrm{M}$ vincristine (Sigma, St. Louis, MO) for $5 \mathrm{hr}, 5 \mu \mathrm{M}$ latrunculin A (Biomol Research Laboratory, Plymouth Meeting, PA) treatment for 24 $\mathrm{hr}$, or $0.03 \%$ DMSO (Sigma) treatment for $24 \mathrm{hr}$ before they were fixed for immunofluorescent light microscopy. STI571/CGP15748 (or Gleevec/imatinib mesylate) kindly was provided by Novartis (Zurich, Switzerland) and was used to inhibit Abl family tyrosine kinases. AG1296, a selective inhibitor for platelet-derived growth factor (PDGF) receptor, was obtained from Calbiochem (La Jolla, CA). Y-27632 treatment $(10 \mu \mathrm{M})$ for $18 \mathrm{hr}$ (Calbiochem) was used to achieve specific inhibition of ROCK.

For double-labeling experiments the neurons were treated with $0.2 \%$ Triton X-100 for 15 min, blocked with 10\% BSA, and stained with rhodamine or FITC phalloidin (Molecular Probes). Antibodies (polyclonal K12 and monoclonal 24-11, Santa Cruz Biotechnology, Santa Cruz, CA; monoclonal Ab-3, Oncogene, Bedford, MA) directed against c-Abl were used to visualize cells transfected with the $v$-abl construct. Alternately, cells were immunostained with monoclonal anti-microtubuleassociated protein 2 (MAP2; Sigma), monoclonal anti-Tau (Tau-1, Sigma), or polyclonal anti-CD71/transferrin receptor (Santa Cruz Biotechnology). After PBS washes the coverslips were mounted and analyzed under either a Zeiss Axiovert S100 or a Zeiss LSM 510 confocal laserscanning light microscope (Carl Zeiss, Thornwood, NY). The morphometric analyses were performed with the MetaMorph 4.6 Imaging software system (Universal Imaging, West Chester, PA).

Glutathione S-transferase pull-down assays. Glutathione S-transferase (GST) pull-down assays were performed as described (Benard et al., 1999; Ren et al., 1999). Briefly, 5 DIV cortical or hippocampal cultures were treated with $0.03 \%$ DMSO for $48 \mathrm{hr}, 3 \mu \mathrm{M}$ STI571 for $48 \mathrm{hr}, 10 \mathrm{nM}$ Toxin B (Calbiochem) for $24 \mathrm{hr}, 100 \mu \mathrm{M}$ hydrogen peroxide for $10 \mathrm{~min}$, or $100 \mu \mathrm{M}$ L-glutamate (Sigma) for $10 \mathrm{~min}$. GTP-bound RhoA, Rac1, and Cdc42 were affinity-precipitated, using the RhoA-binding domain of Rhotekin (GST-RBD) or the Rac1/Cdc42-binding domain of PAK (GSTPBD; Cytoskeleton, Denver, CO). Bound proteins were washed, resolved on $8-16 \%$ SDS-PAGE gels, transferred to PVDF membranes (Millipore, Bedford, MA), and immunoblotted with monoclonal anti-RhoA (Cytoskeleton), monoclonal anti-Rac1 (BD Transduction Laboratories, Lexington, KY), or polyclonal anti-Cdc42 (Cytoskeleton), using enhanced chemiluminescence (Amersham Biosciences, Piscataway, NJ) for detection. Densitometric analysis of films was performed with the Bio-Rad GelDoc Imaging system (Bio-Rad Laboratories, Hercules, CA). Relative amounts of GTP-bound RhoA, Rac1, or Cdc42 for each treatment were determined by measuring the amount of RhoA, Rac1, or Cdc42 sedimented by the GST-RBD or GST-PBD relative to the total amount of RhoA, Racl, or Cdc42 in the control (DMSO) treatment.

Time-lapse light microscopy. To determine the dynamics of dendrogenesis of a single neuron, we have applied time-lapse light microscopy according to a procedure described earlier (Jones et al., 2002; Kim et al., 2002). Briefly, dissociated hippocampal neurons treated with DMSO as a control or STI571 for $2 \mathrm{~d}$ from 5 to 7 DIV were grown on $25 \mathrm{~mm}$ coverslips and placed in an Attofluor cell chamber (Atto Instruments, Rockville, $\mathrm{MD}$ ) on the heated stage of a Zeiss Axiovert microscope, which was supported by an anti-vibration table. Differential interference contrast images at $63 \times$ were captured by using a Hamamatsu Orca camera (Bridgewater, NJ). To minimize phototoxicity for the living cells, we used a computer-driven automatic shutter to achieve minimum illumination (300 msec/frame). The longest continuous recording was $135 \mathrm{~min}$. For some experiments, at the end of the time-lapse recording the cells were fixed and processed for Hoechst 33258 nuclear staining and anti-MAP2 immunocytochemistry to evaluate the status of neuronal cell death. The integrity of nuclear and cell morphology as well as dendritic motility indicated that the neurons were healthy throughout the incubation period. For some time-lapse experiments the neurons first were grown in normal medium for $7 \mathrm{~d}$ and then were placed on stage for recording. DMSO or STI571 was added for 135 min to examine the onset of dendritic deterioration caused by STI571.

In some experiments, low-density hippocampal neurons (5000/25 $\mathrm{mm}^{2}$ ) were cultured on etched-grid coverslips (Bellco, Vineland, NJ) coated with poly-L-lysine. For this type of experiment we typically took images of $\sim 3-5$ neurons per coverslip at $0.5,3,4,5,6$, and 7 DIV. After the neurons were imaged, they were returned to the incubator. Dishes were kept outside the incubator for $<20 \mathrm{~min}$ on the heated stage. The etchings on the coverslips made it possible to identify the same neurons on subsequent days.

Data analysis. We used MetaMorph 4.6 (Universal Imaging) to analyze the single cell images from neurons grown on the etched coverslips and immunofluorescent light microscopy of neurons cultured on $25 \mathrm{~mm}$ coverslips or from the recorded time-series of neurons grown in the Attofluor chamber. Dendrite numbers were determined by using Measure Count Object function. Primary dendrites were considered when processes extended directly from the cell body, and they were scored when they were longer than $10 \mu \mathrm{m}$. Secondary dendrites were considered when processes branched off primary dendrites, and they were scored when they were longer than $10 \mu \mathrm{m}$. Processes shorter than $10 \mu \mathrm{m}$ were considered as sprouts. To analyze the axon and dendrite indexes (length and number of branches) on neurons grown on the etched coverslips at $25 \times$ magnification, we used the total length of dendrites and their numbers. To analyze the relative dendritic complexity affected by different treatments, we determined the dendrite number and length in a bracketed area of $120 \mu \mathrm{m}^{2}$ around the neurons imaged at $63 \times$ magnification. For each dendrite measurement the sprouts and branches along the 80 $\mu \mathrm{m}$ length of a given dendrite were scored in total of 30 neurons from at least three independent experiments. Dendrite length was determined by using Region Measurement function. Numerical values of number and length measurements were logged onto Microsoft Excel (Redmond, WA) automatically. For some analyses, when it was necessary to pool dendrite length measurements from different experiments, their values were normalized. The histories of dendrite elongation and retraction were produced by Microsoft Excel and SigmaPlot 8.0 (SPSS Science, Chicago, IL). 
For immunofluorescent light microscopy of latrunculin A-treated neurons, F-actin was fragmented, and the staining was weak. The images were enhanced intentionally to reveal dendritic morphology for dendrite number and length measurement. The F-actin staining was also weak for jasplakinolide-treated neurons because of its competition with fluorescent phalloidin for F-actin binding. The images were enhanced similarly to reveal dendritic morphology for dendrite number and length measurement.

One-way ANOVA and Dunnett's procedure for means were performed, and $p$ values were assigned in each experiment. Any null hypothesis with the probability level $<95 \%$ was rejected.

\section{Results \\ Inhibition of Abl family tyrosine kinases disrupts dendrogenesis independently of axonogenesis}

Hippocampal neurons acquire their polarized morphology in a well characterized sequence of events (Dotti et al., 1988). To examine what role the Abl family tyrosine kinases may play during dendrogenesis, we compared the developmental progression of primary hippocampal neurons with those cultures treated with $3 \mu \mathrm{M}$ STI571 (CGP15748/Gleevec/imatinib mesylate), a well known anti-cancer drug widely used to treat chronic myelogenous leukemia. It is an established small molecule inhibitor of Abl family tyrosine kinases that include both Abl and Arg (Ablrelated gene) kinases (Buchdunger et al., 1996; Carroll et al., 1997; Lu et al., 2002). STI571 also shows an inhibitory effect on the PDGF receptor tyrosine kinase in fibroblast cells (Buchdunger et al., 2000).

In control cells the typical formation of lamellipodial structures from the spherical cell body occurs by 0.5 DIV (Fig. $1 A$ ). This is followed by the extension of multiple neurites, one of which assumes axonal identity, by 3 DIV. Between this point and 7 DIV, dendritic identity is established, and dendritic processes elongate and become more structurally complex while axonal outgrowth continues independently.

To examine the effects of Abl family kinase inhibition during dendrogenesis, we applied STI571 to hippocampal cultures between 4.5 and 7 DIV. This allowed us to examine the effects of Abl family kinase inhibition on dendrogenesis after the axonal identity had been established fully. After $24 \mathrm{hr}$ STI571 treatment resulted in a reduction of dendrite length as well as a corresponding attenuation of branch formation (Fig. $1 \mathrm{~B}$, arrows point to dendrites; arrowheads point to the axon). This trend continued through 6 DIV, when dendrite length and branch formation both were decreased markedly. By 7 DIV dendritic development was inhibited, and degeneration was apparent (Fig. $1 B$, arrows). We also noted the appearance of cell body rounding, an effect of Rho activation in a number of different cell types, including neuronal cell lines (Leeuwen et al., 1997; Kranenburg et al., 1999). The
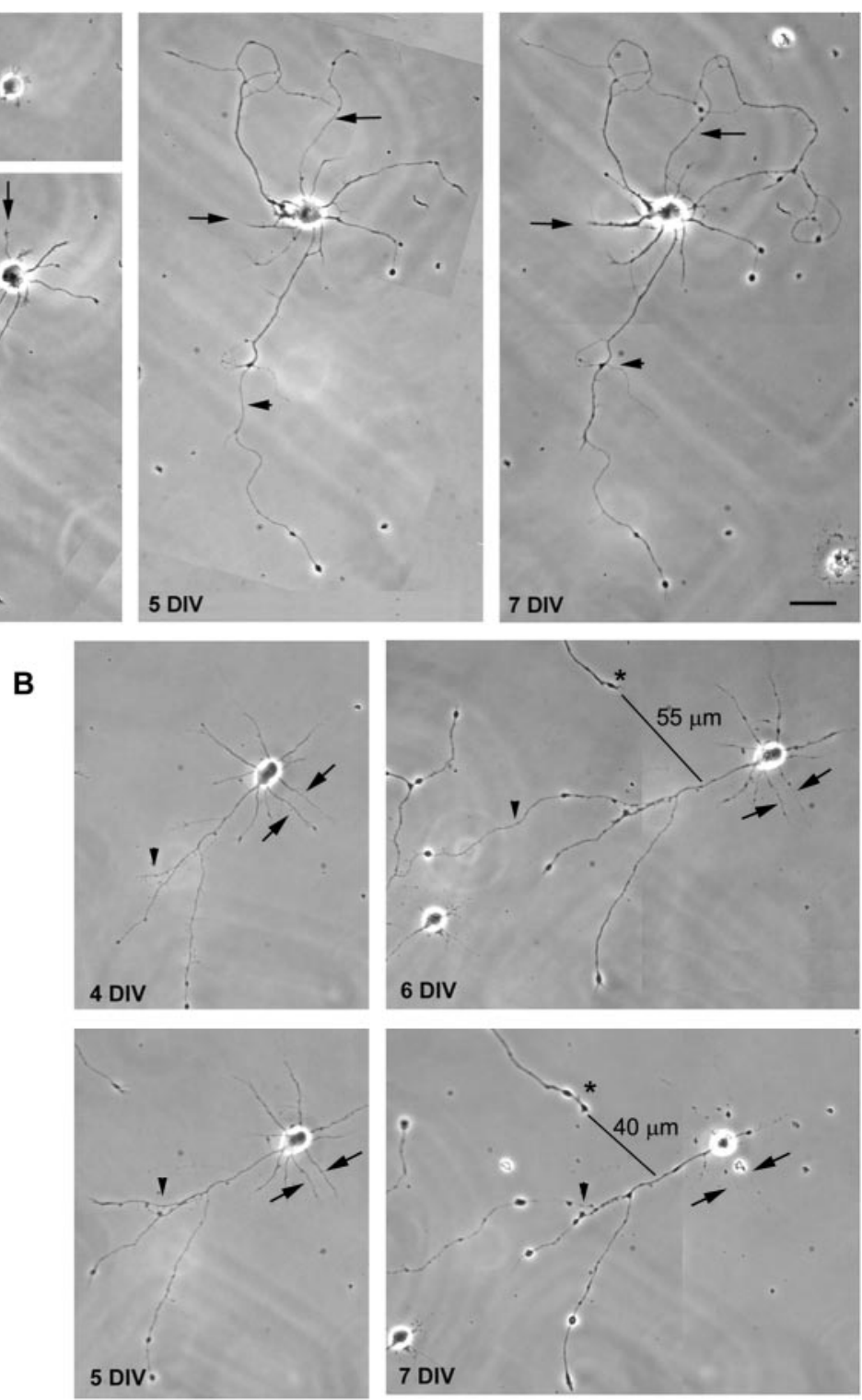

Figure 1. Inhibition of Abl family tyrosine kinase activity disrupts dendrogenesis independently of axonogenesis. $A$, Developmental progression of cultured hippocampal neurons on etched-grid coverslips from 0.5 through 7 DIV. $B$, Treatment with the small molecule inhibitor STI571 from 4 DIV impairs dendrogenesis while axons continue to grow and branch. Arrows point to dendrites; arrowheads indicate axons. The asterisks indicate an approaching axon on 6 versus 7 DIV. Scale bar, $25 \mu \mathrm{m}$.

effects of STI571 on dendrogenesis were specific for Abl family tyrosine kinases, because treatment with $5 \mu \mathrm{M}$ AG1296 to inhibit PDGF receptor tyrosine kinase $\left(\mathrm{IC}_{50}=0.5 \mu \mathrm{M}\right)$ did not show any effects on dendrogenesis. The effects of STI571 on dendrogenesis were dose-dependent, because $60 \mathrm{~nm}$ had little impact whereas maximum inhibition was achieved at $5 \mu \mathrm{M}$ treatment. In addition, STI571 effects were limited to actively developing dendritic structures and were reversible, because dendrogenesis resumed and showed no significant difference by 10 DIV when STI571 was withdrawn on 7 DIV (data not shown). We noted that Abl kinase inhibition had a less striking effect on axon morphology after establishment of axonal identity. Axon growth and branch formation continued in both control and STI571 treatments (Fig. $1 A, B)$. On $7 \mathrm{DIV}$, although there were no significant differences in axon length and branch points between control and STI571treated neurons, STI571 led to a remarkably reduced dendritic number and length (Table 1). On the other hand, overexpression 
Table 1. Effects of Abl tyrosine kinases on neuronal morphology

\begin{tabular}{lrr}
\hline Neuronal morphology & Control & STI571 \\
\hline Axon length (in micrometers) (per neuron) & $100.00 \pm 13.60$ & $108.26 \pm 14.70$ \\
Axon branch (\#) (per neuron) & $100.00 \pm 10.40$ & $86.40 \pm 15.60$ \\
$1^{\circ}$ dendrite length (in micrometers) (per neuron) & $100.00 \pm 2.78$ & $85.22 \pm 3.47^{*}$ \\
$2^{\circ}$ dendrite length (in micrometers) (per neuron) & $100.00 \pm 4.48$ & $86.47 \pm 8.43$ \\
$1^{\circ}$ dendrite (\#) (per neuron) & $100.00 \pm 6.73 \pm 5.29^{*}$ \\
$2^{\circ}$ dendrite (\#) (per neuron) & $100.00 \pm 6.98$ & $114.05 \pm 3.88^{*}$ \\
Nuclear condensation & $100.00 \pm 13.30$ & $95.00 \pm 4.57$ \\
\hline
\end{tabular}

A total of 18 neurons from at least two independent transfection experiments were analyzed for their axon and dendrite number and length. A total of 40 neurons were scored for nuclear condensation using Hoechst staining. Each numbe represents the percentage (mean \pm SEM) of DMSO as the control. * ${ }^{*}$ ignificantly different from control, $p<0.05$.

of a constitutively active $\mathrm{Abl}(\mathrm{CA}-\mathrm{Abl})$ resulted in an overall increase in dendritic complexity (dendritic branching and extension), especially the primary dendrite number and length (Table 1). These data indicate that Abl family kinases play an important role in the formation of dendritic arbor and that this involvement may be separate from axonogenesis.

\section{Inhibition of Abl family tyrosine kinase activity alters the developmental dynamics of dendrogenesis}

To examine the dynamics of dendrogenesis influenced by $\mathrm{Abl}$ kinase inhibition, we used time-lapse microscopic imaging. Control neurons treated with DMSO at 7 DIV displayed rapid motility of primary and secondary dendrites as well as extension and retraction of dendritic sprouts. We designated the primary dendrites as those processes longer than $10 \mu \mathrm{m}$ that extended directly from the neuronal cell body. Secondary dendrites were those that branched off directly from the primary dendrites.

Because the major measurable changes in dendritic dynamics of neurons treated with DMSO or STI571 in 7 DIV neurons were proximal to the cell body, we focused on the dendrite activities within the $120 \mu \mathrm{m}^{2}$ brackets. After a 135 min recording experiment the control dendrite length increased significantly (Fig. $2 \mathrm{~A}$, asterisks point to the extension of one such dendrite). During this time period an individual dendrite can elongate and retract for a few minutes, followed by a major advance (Fig. 2 B). For example, two major advances can be demonstrated in the average life history of 25 dendrites (Fig. 2B, DMSO at 40 and $90 \mathrm{~min}$, respectively). At 7 DIV primary dendrites extended at a slower rate, giving a $22 \%$ increase in length, whereas secondary dendrites elongated rapidly, leading to a $44.8 \%$ increase in length at the end of the recording period (Fig. $2 C$, DMSO). In contrast, although individual secondary dendrites in neurons treated with STI571 from 5 to 7 DIV showed a modest elongation (Fig. $2 \mathrm{~A}$, arrowhead), the length of most dendrites remained unchanged or was decreased slightly (Fig. $2 \mathrm{~A}$, number symbol points to a retracting dendrite). This resulted in no overall elongation of primary dendrites. Secondary dendrites showed a small $(9.8 \%)$ but insignificant increase in length (Fig. $2 B, C$ ). We also evaluated dendritic sprout formation in DMSO- and STI571-treated neurons as a measure of dendritic complexity. Although dendritic sprout numbers remained unchanged in both control and STI571treated neurons before and after recording, there were significantly fewer sprouts in STI571-treated neurons when compared with control cells (Fig. 2A,D). Thus, although sprout formation did occur under conditions of Abl kinase inhibition during the 135 min time-lapse recording, additional extension was limited, leading to a net retraction and subsequent reduction in dendrite complexity (Fig. $2 A$ ). As with previous experiments, no significant differences in axonal outgrowth were noted in response to Abl kinase inhibition after axonal establishment. Under this experimental condition there were no changes in lamellipodia mo- tility of growth cone dynamics in DMSO- or STI571-treated neurons (Fig. $2 A$, arrowheads) (for additional information on growth cone and dendrite dynamics by time-lapse video light microscopy, see supplemental material, available at www.jneurosci.org). These experiments suggest that Abl kinase inhibition may impair the function of molecular machinery required for dendritic branching, which is highly active at 7 DIV.

\section{Inhibition of Abl family tyrosine kinases disrupts axon-dendrite polarity}

To determine whether the cytoskeletal organization of hippocampal neurons is altered by the inhibition of Abl tyrosine kinases, we performed double-immunofluorescent light microscopy. At 7 DIV the control neurons showed an elaborate dendritic arbor, which was supported by the underlying actin network (Fig. 3Aa, arrows). Microtubule-associated protein 2 (MAP2), which is specific for dendrite localization, was clearly present in the dendrites (Fig. 3Ab, arrows) but excluded from the axon (Fig. 3Ab, arrowhead). Microtubule-associated protein Tau, known to segregate into the axonal compartment, was present in the axon (Fig. $3 A c$, arrowhead) but only weakly visible in the dendrites (Fig. 3Ac, arrows). However, neurons treated with STI571 from 4.5 to 7 DIV showed localized enrichment of actin filament at the growth cones of axons (Fig. $3 \mathrm{Ba}$, asterisk) and dendrites (Fig. 3Ba, arrows). A weak anti-MAP2 immunostaining was seen in the dendrites, but it also became positive in the axon, indicating the failure of MAP2 segregation (Fig. $3 \mathrm{Bb}$, arrows indicate dendrites; arrowhead points to the axon). The disruption of normal axon-dendrite polarity was evident, because Tau protein not only was present in the axon (Fig. $3 B c$, arrowhead) but also was observed in the dendrites (Fig. $3 B c$, arrows).

To determine whether the disruption of dendritic polarity is MAP2-specific, we examined the distribution patterns of CD71 (transferrin receptor) and the glutamate receptor, two other well established dendritic markers in hippocampal cultures (West et al., 1997; Li et al., 1998). At 7 DIV the MAP2 and the transferrin receptor showed overlapping staining patterns in control neurons (Fig. 4, DMSO, MAP2, and CD71 arrows). When 5 DIV neurons were treated with STI571 for $48 \mathrm{hr}$, the transferrin receptor was codistributed with MAP2 in all processes, and dendritic deterioration was obvious (Fig. 4, STI571, MAP2, and CD71). NR2A, a regulatory subunit of the NMDA subtype ionotropic glutamate receptor, began to express and localize to the dendrites of 7 DIV hippocampal neurons (Fig. 4, DMSO, NR2A arrow). This can be confirmed by double-labeling neurons with anti-Tau, which showed a complementary staining pattern to NR2A (Fig. 4, DMSO, Tau arrowhead). When neurons were treated with STI571 for $48 \mathrm{hr}$, NR2A and Tau were present in all of the processes (Fig. 4, STI571, NR2A, Tau). The phenotype for a decrease in dendrite number and length in neurons immunostained with 
A

DMSO
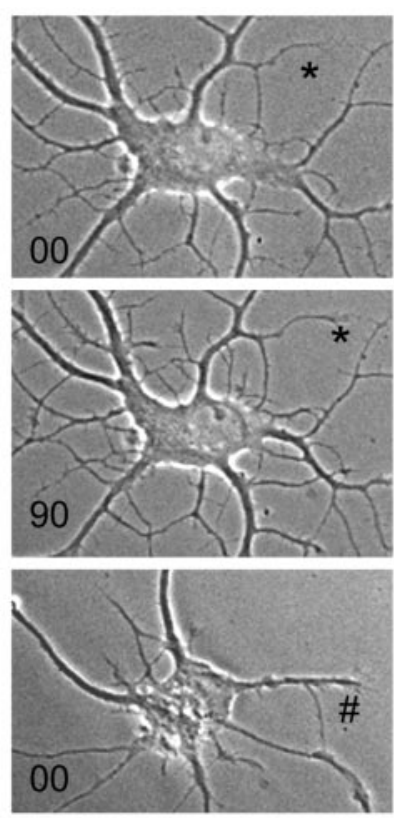

STI571

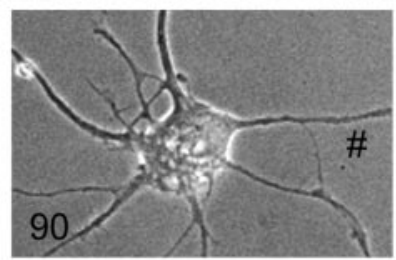

B

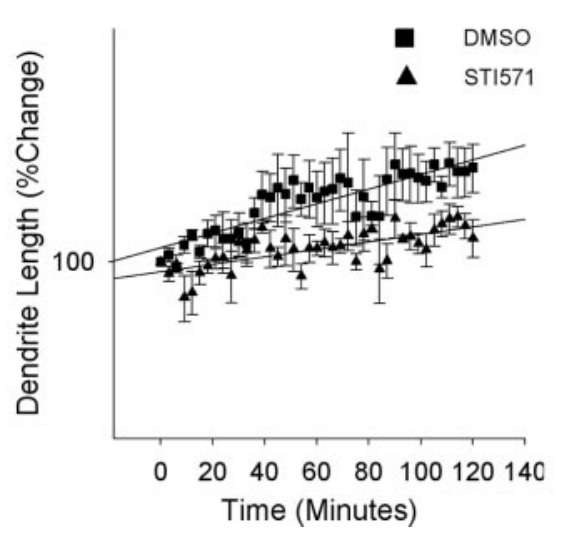

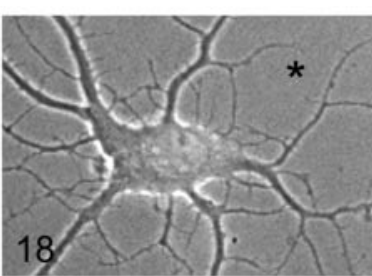
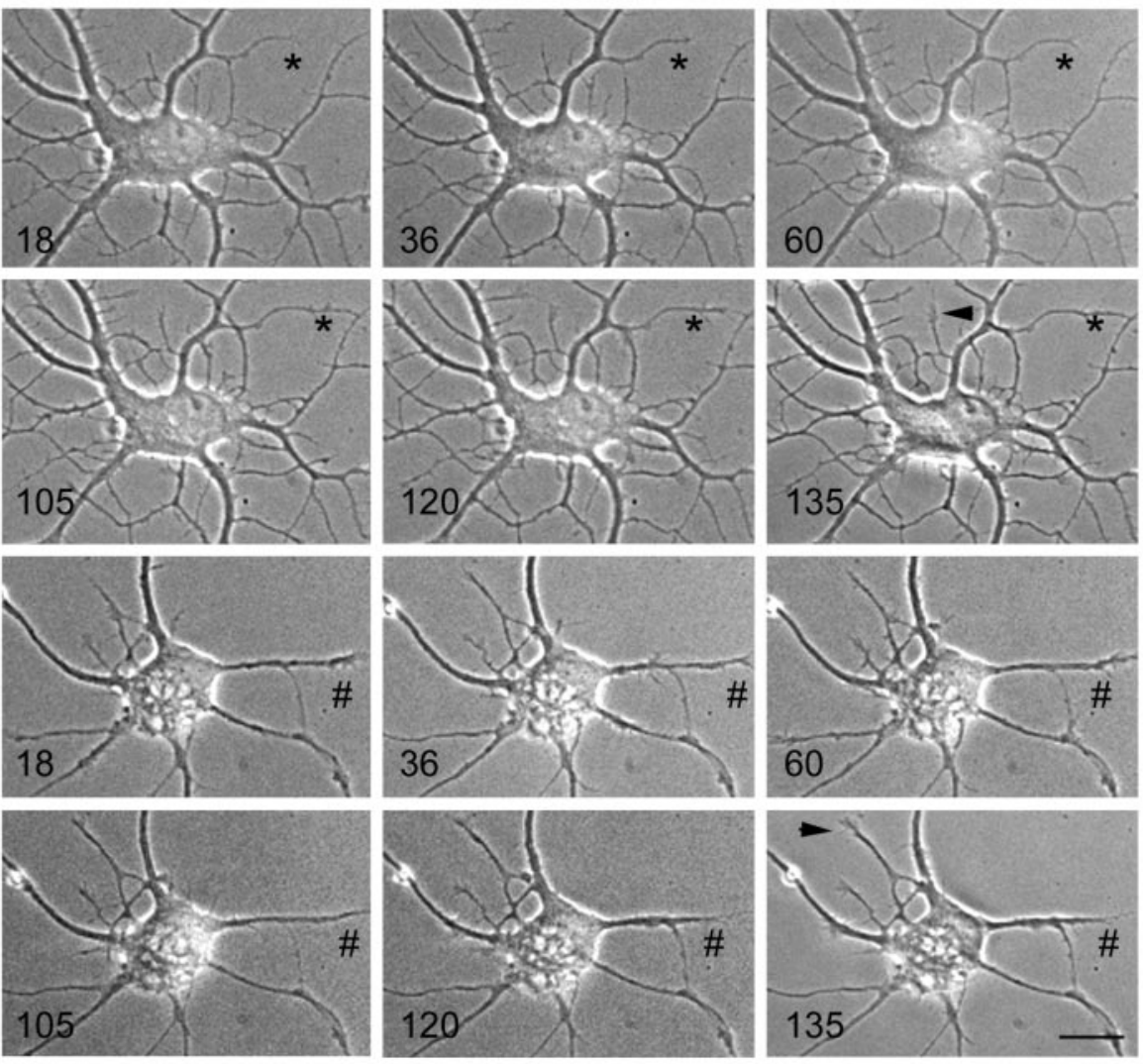

D
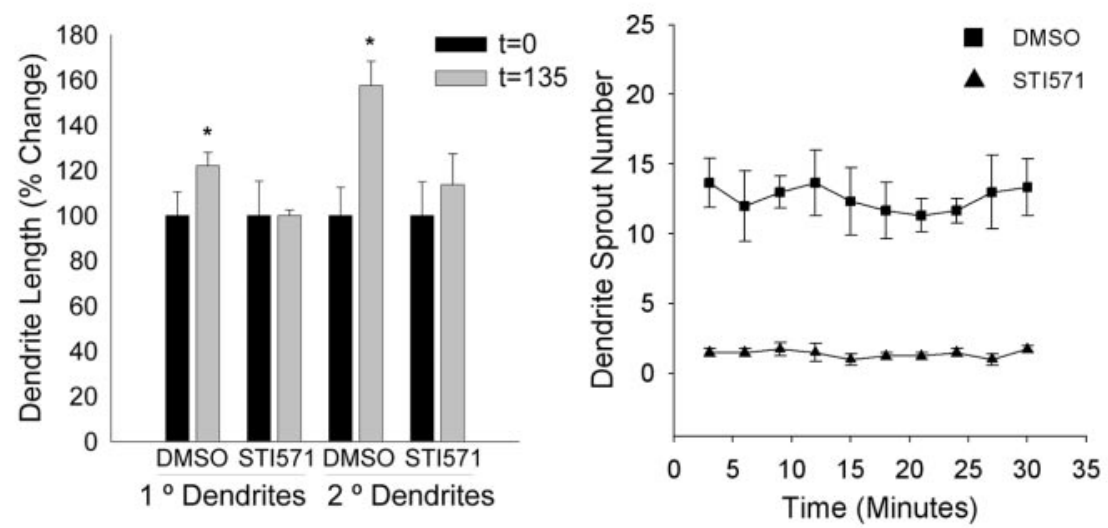

Figure 2. Inhibition of Abl family tyrosine kinase activity alters the developmental dynamics of dendrogenesis. A, Hippocampal neurons grown in culture for $4.5 \mathrm{~d}$ were incubated in the absence (DMSO) or presence (STI571) of Abl inhibitor for $48 \mathrm{hr}$. Time-lapse images were captured every 30 sec for $135 \mathrm{~min}$. Only eight representative frames are shown here. Asterisks point to an elongating dendrite in neurons treated with DMSO. The number signs point to a retracting dendrite in neurons treated with $3 \mu \mathrm{M}$ STI571. The arrowhead points to growth cones in neurons treated with DMSO or STI571. Scale bar, $15 \mu \mathrm{m}$. B, Comparison of histories of the percentage of change in dendrite length of neurons treated with DMSO and STI571. Shown here are the measurements of average length changes in 25 dendrites during a 135 min time-lapse recording. C, Comparison of the percentage of change in primary and secondary dendrite length of neurons treated with DMSO and STI571. Shown here are the measurements of average length changes in 25 dendrites before and after time-lapse recording. Asterisks indicate the significant differences between $t=0$ and $t=135$ min $\left({ }^{*} p<0.05\right)$.D, Comparison of the histories of dendritic sprout formation between DMSO-and STI571-treated neurons during 30 min time-lapse recordings. In this case, sprouts were measured along $80 \mu \mathrm{m}$ length of a dendrite, and a total of seven dendrites was selected for analysis from time-lapse light microscopy. Error bars indicate SEM. For details, see supplemental material, available at www.jneurosci.org.

anti-transferrin receptor and anti-NR2A was similar to degenerating neurons stained with anti-MAP2. This result showed that STI571-induced dendritic degeneration is not limited to the alteration of MAP2 but that the distribution and segregation of other dendritic-specific markers also are affected.
Abl family tyrosine kinase activity influences primary dendrite formation and secondary dendritic branching To examine further the effect of Abl kinase activity on morphological features of dendrite formation, we quantified the dendritic characteristics, such as primary dendrite formation and 


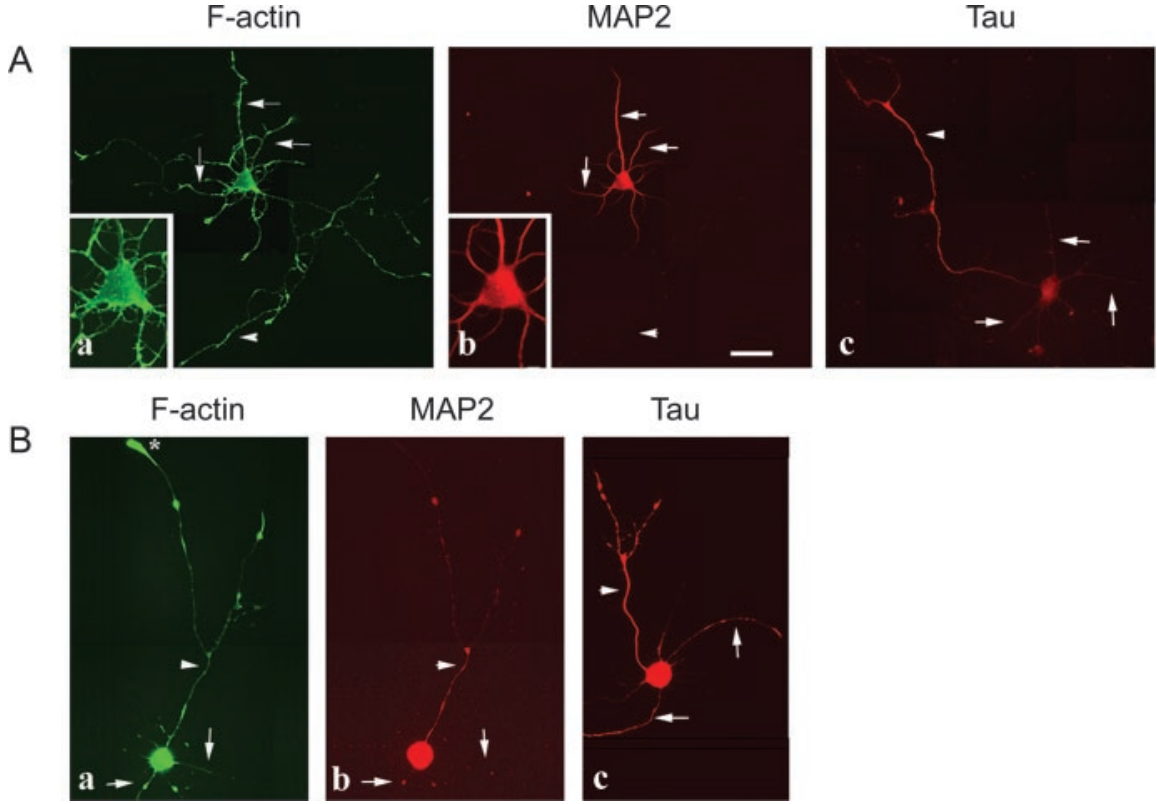

Figure 3. Inhibition of Abl family tyrosine kinase activity disrupts axon-dendrite polarity. $A$, Immunofluorescent images of control neurons labeled with FITC phalloidin ( $a)$, anti-MAP2 (b), and anti-Tau (c). B, Hippocampal neurons at 4.5 DIV treated with $3 \mu \mathrm{M}$ STI571 for $48 \mathrm{hr}$, stained with FITC phalloidin ( $a$ ), and immunostained for anti-MAP2 (b) and anti-Tau (c). Note disruption of MAP2 and Tau segregation after Abl kinase inhibition. Arrows point to dendrites; arrowheads indicate axons. Asterisk points to the growth cone. Scale bar, $25 \mu \mathrm{m}$.

secondary dendrite branching, in response to Abl kinase inhibition and overexpression of CA-Abl (Fig. 5). All quantification was based on MAP2-positive staining to differentiate dendritic processes. Phase-contrast and immunofluorescent light microscopy of single neurons showed that the endogenous Abl expression was present throughout the neuronal cell body and the processes (Fig. 5A, DMSO and STI571), although the immunostaining was weak when compared with the intensive Abl immunoreactivity of the cells transfected with CA-Abl (Fig. $5 A, \mathrm{CA}-\mathrm{Abl})$. STI571 treatment reduced the number of MAP2positive primary dendrites when compared with DMSO-treated neurons (Fig. 5A, DMSO and STI571). In contrast, CA-Abl overexpression induced an exuberant formation of MAP2-positive dendrites (Fig. 5A, CA-Abl). To quantify the number of dendrites per neuron and measure the length of dendrites, we applied the Region Function of MetaMorph imaging software. Dendrites within the area of $120 \mu^{2}$ were scored. When compared with the control, Abl inhibitor-treated neurons showed a $42 \%$ reduction in primary dendrite number and $45 \%$ reduction in secondary dendrite number per cell (Fig. $5 B$ ). This is in contrast to neurons overexpressing $\mathrm{CA}-\mathrm{Abl}$, in which the primary dendrite number increased by $20 \%$ (Fig. $5 B$ ). The effects of CA-Abl overexpression appeared to influence primary dendrites more dramatically, because the number of secondary dendrites in these neurons did not differ significantly when compared with control neurons (Fig. 5B). We also examined the effect of Abl kinase activity on the relative length of primary and secondary dendrites (Fig. 5C). Compared with control neurons, STI571-treated neurons showed a modest decrease in primary dendrite length by $15 \%$ and secondary dendrite length by $17 \%$. CA-Abl overexpression led to an increase in primary dendrite length by $15 \%$ but had little effect on secondary dendrite length. In addition, the number of dendrites may be overestimated slightly in STI571-treated neurons because, in some neurons, axons could be mistaken for dendrites as they became MAP2-positive (Fig. 3B). These analyses demonstrated that a bal- anced Abl tyrosine kinase activity is important in maintaining a physiological pace of dendrogenesis.

Inhibition of Abl family tyrosine kinase activity leads to an increase in GTPbound RhoA without affecting Racl or Cdc42 activity

The morphological features observed with modulation of Abl kinase activity are reminiscent of those induced by the Rho family of small GTPases, which are important regulators of many aspects of neurogenesis, including dendrogenesis. RhoA has been reported widely to regulate dendrite outgrowth negatively, whereas Racl and Cdc42 have been shown to increase dendrite length and complexity (Katoh et al., 1998; Lee et al., 2000; Luo, 2000; Nakayama et al., 2000). To determine whether the activity of Rho family small GTPases is affected by the inhibition of Abl kinase activity, we used an affinity precipitation technique to quantitate active (GTP-bound) Rho GTPases (Benard et al., 1999; Ren et al., 1999). Primary neuronal cultures were subjected to a series of pharmacological treatments before evaluation of Rho GTPase activity. When 5 DIV neurons were treated with DMSO as a control for $48 \mathrm{hr}$, a basal level of active RhoA, Rac1, and Cdc42 could be detected (Fig. 6A, right, DMSO). STI571 treatment increased RhoA activity but did not induce significant changes in Rac1 or Cdc42 activity (Fig. 6A, right, STI571). Toxin $B$, a well established inhibitor of Rho GTPase activity, suppressed GTP-bound RhoA, Rac1, and Cdc42 (Fig. 6A, right, Toxin B). We treated neurons with STI571 in the presence of hydrogen peroxide as a positive control because it additionally enhanced RhoA activity (Fig. 6 A, right, STI571 plus $\mathrm{H}_{2} \mathrm{O}_{2}$ ). Glutamate treatment leads to actin remodeling in dendritic spines, and the stimulus-induced dendritic arbor growth requiring glutamate receptor-mediated synaptic transmission has been shown to increase Rac1 and Cdc42 activity (Fischer et al., 2000; Jones et al., 2002; Sin et al., 2002; Portera-Cailliau et al., 2003). We therefore subjected neurons at 7 DIV to $100 \mu \mathrm{M}$ glutamate for $10 \mathrm{~min}$. Compared with DMSO- or STI571-treated neurons, a brief treatment of glutamate induced an activation of Rac1, but not Cdc42 (Fig. 6A, right, glutamate). As a control, 7 DIV cell lysates with different treatments were probed with anti-RhoA, Rac1, or Cdc42 to show that the protein expression of these GTPases did not change (Fig. $6 \mathrm{~A}$, left). Semiquantification using Bio-Rad GelDoc showed that STI571 resulted in a 30\% increase in RhoA activity, whereas the changes in Racl and Cdc42 activity were insignificant (Fig. 6B). Taken together, these data indicate that RhoA may be a downstream target of $\mathrm{Abl}$ kinase activity, whereas Racl and Cdc42 may not be targeted directly in 7 DIV hippocampal neurons.

\section{Rho kinase inhibition suppresses the inhibitory effects of STI571 on dendrogenesis}

Although RhoA has many downstream effectors, Rho-associated kinase or p160/ROCK has been shown to be particularly important in neuronal morphogenesis. This is demonstrated in several studies that have shown that RhoA-mediated neurite retraction can be prevented by inhibition of ROCK activity (Hirose et al., 
DMSO
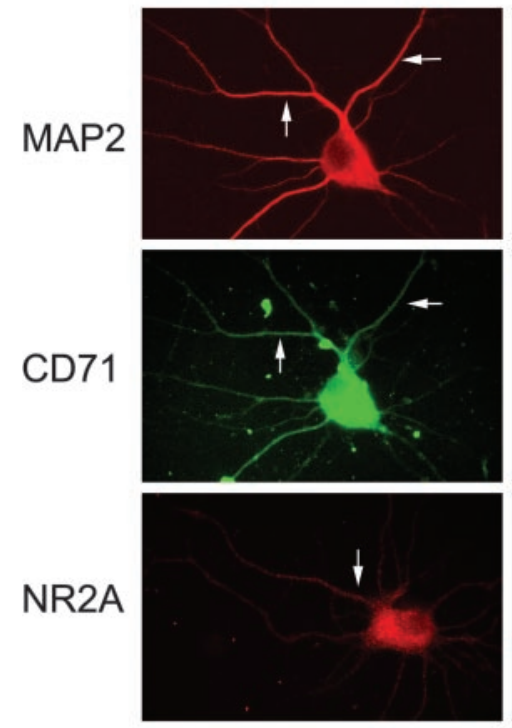

Tau
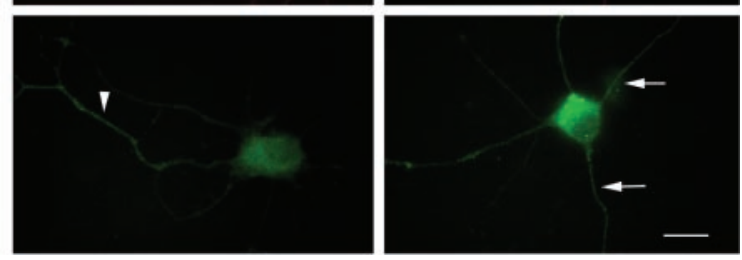

Figure 4. Immunofluorescent images of hippocampal neurons at 5 DIV treated with $0.03 \%$ DMSO (left) or $3 \mu$ STI571 (right) for $48 \mathrm{hr}$ and double-immunolabeled with anti-MAP2 and anti-CD71 (transferrin receptor) or anti-NR2A and anti-Tau. Arrows point to the dendrites; arrowhead points to the axon. Note the colocalization of MAP2 with CD71 in DMSO- and STI571treated cells. Also note that, whereas Tau was localized to the axon, NR2A was localized more prominently in dendrites (arrow), with weaker staining in the axon (DMSO; compare Tau and NR2A staining). The segregation of Tau and NR2A was not clear in neurons treated with STI571. Scale bar, $15 \mu \mathrm{m}$.

1998; Bito et al., 2000; Nakayama et al., 2000). Because morphological and biochemical data suggest an association of the Abl family kinases and RhoA signaling, we speculated that ROCK also may lie downstream of Abl. To examine this more closely, we used Y-27632, a ROCK-specific inhibitor (Uehata et al., 1997), to determine whether the STI571-induced dendritic simplification can be reversed when ROCK activity is blocked. Doubleimmunofluorescent light microscopy of single neurons demonstrated that 7 DIV neurons showed an elaborate MAP2-positive dendrite formation and displayed a well defined F-actin network (Fig. 7A, STI571/- and Y-27632/-). STI571 treatment suppressed dendritic elaboration, which is characterized by a reduced number of MAP2-positive dendrites. The fluorescent phalloidin staining showed an increased focal F-actin organization (Fig. 7A, STI571/+ and Y-27632/-, arrowheads), although its overall intensity was decreased in dendrites (Fig. 7A, STI571/+ and $\mathrm{Y}-27632 /-$ ) (here the image of F-actin staining was enhanced to show dendritic number and length). Neurons treated with $10 \mu \mathrm{M}$ Y-27632 alone resulted in an increase in dendrite length (Fig. 7A, STI571/- and Y-27632/+). When it was compared with STI571 treatment alone, STI571 in the presence of Y-27632 elicited a reversal of dendritic simplification toward the level of arborization seen in control cells (Fig. 7A, STI571/+ and $\mathrm{Y}-27632 /+)$.

When the effect of Y-27632 on dendrogenesis was quantified, it became clear that, although STI571 inhibited both the numbers of primary and secondary dendrites, Y-27632 was able to suppress the effect of STI571 (Fig. 7B). Y-27632 treatment alone did not affect the number of primary and higher order dendrites significantly. STI571 also reduced the length of both primary and secondary dendrites when compared with neurons treated with DMSO as the control (Fig. 7C). Y-27632 treatment alone, however, increased the dendrite length dramatically. Neurons treated with Y-27632 reversed the inhibitory effect of STI571 on the length of secondary dendrites but additionally promoted the length of primary dendrites when compared with neurons treated with DMSO. The ability of Y-27632 to reverse STI571 effects on dendrites was rather extensive, because Y-27632 was applied to neurons previously treated with STI571 for $24 \mathrm{hr}$. Thus our results indicate that $\mathrm{Abl}$ kinase activity indeed plays a critical role in the upstream regulation of both RhoA and its downstream effector ROCK during dendrogenesis.

\section{RhoA activation suppresses CA-Abl effect on dendrogenesis} In addition to the simplification of the dendritic tree that was observed with the inhibition of the Abl family kinases, we also found a reciprocal increase in dendritic complexity with overexpression of a constitutively active Abl kinase (Fig. 5). If Abl kinase activity negatively regulates RhoA, it stands to reason that expression of constitutively active RhoA may be sufficient to block the phenotype seen with Abl kinase activation. To test this hypothesis, we coexpressed CA-Abl and constitutively active RhoA (CARhoA) in hippocampal neurons (Fig. 8). Constitutive activation of Abl kinase induced an exuberant formation of dendritic arbors (Fig. 8, pEGFP-C2/+ and CA-Abl/+) (also Fig. 5A, CA-Abl). Consistent with previous reports, CA-RhoA was sufficient to induce dendrite retraction and resulted in a drastic simplification of the dendritic arbor (Fig. 8, CA-Abl/- and CA-RhoA-GFP/+) (Nakayama et al., 2000). When CA-RhoA and CA-Abl were coexpressed, we observed a complete reversal of the CA-Abl phenotype to that seen with CA-RhoA alone (Fig. 8, CA-Abl/+ and $\mathrm{CA}-\mathrm{RhoA}-\mathrm{GFP} /+$ ). Overall, these data indicate that the activity of Abl kinase plays a critical role in the regulation of dendrite elaboration during dendrogenesis via a RhoA/ROCK-dependent mechanism.

\section{The CA-Abl effect on dendrogenesis is dependent on the} reorganization of the actin cytoskeleton but is independent of microtubule stability

Modulation of Abl kinase activity appears to signal through RhoA and ROCK, both of which have been shown to be essential in the regulation of actin stability and neurite sprouting ( Da Silva et al., 2003). We have speculated that Abl kinase activity also may affect the underlying cytoskeletal structure of primary neurons in association with RhoA/ROCK-dependent signaling. To examine this more closely, we pharmacologically modified the actin cytoskeleton in conjunction with overexpression of CA-Abl kinase to determine whether the CA-Abl phenotype is dependent on reorganization of actin (Fig. 9A). After microfilament destabilization with latrunculin A alone, F-actin was fragmented, and we noted an increase in neuronal process formation, which resembles the phenotype generated by CA-Abl (Fig. 9A, compare $\mathrm{F}$-actin in neurons treated with $\mathrm{CA}-\mathrm{Abl} /+$ and latrunculin $\mathrm{A} /+$ ). Treatment with latrunculin A, in conjunction with CA-Abl overexpression, yielded a phenotype similar to both treatments individually, which indicates the requirement for actin disruption in process formation in response to Abl activation (Fig. 9A, CA$\mathrm{Abl} /+$ plus latrunculin $\mathrm{A} /+$ ). F-actin staining became fragmented because of the effects of latrunculin A treatment. In the 


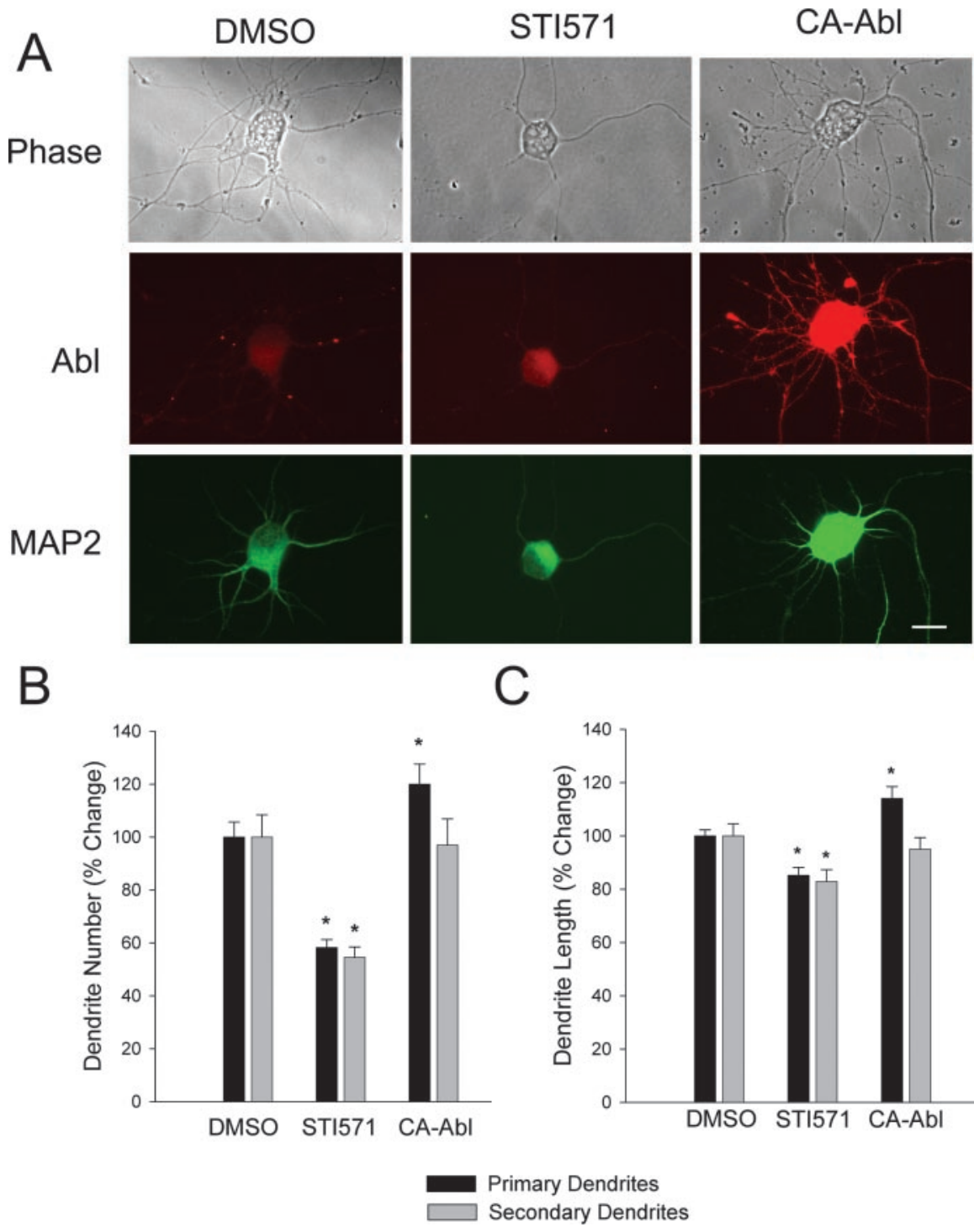

Figure 5. Abl family tyrosine kinase activity influences primary dendrite formation and dendritic branching. $A$, Phase-contrast and double-immunofluorescent light microscopy of single neurons treated with DMSO or STI571 or transfected with CA-Abl. Neurons were treated for $48 \mathrm{hr}$ from 5 to 7 DIV and immunostained with rabbit polyclonal anti-Abl and mouse monoclonal anti-MAP2. Note the simplification of dendritic profile in STI571-treated neurons in comparison to an exuberant outgrowth of MAP2-positive, highly branched processes in neurons transfected with CA-Abl. Scale bar, $15 \mu \mathrm{m}$. B, Quantification of primary and secondary dendrite outgrowth in neurons incubated with DMSO or STI571 or transfected with CA-Abl. C, Quantification of primary and secondary dendrite length in neurons incubated with DMSO or STI571 or transfected with CA-Abl. Error bars indicate SEM; ${ }^{*} p<0.05$.

reverse experiment we used jasplakinolide, a cell-permeable macrocyclic peptide that is a potent inducer of actin polymerization, to determine whether the CA-Abl phenotype can be blocked by stabilization of the actin cytoskeleton. Treatment with jasplakinolide alone resulted in a simplification of dendritic branching (Fig. 9A, jasplakinolide/+). When CA-Abl expression was combined with jasplakinolide treatment, the dendritic branching became less extensive than in neurons expressing CA-Abl without jasplakinolide treatment (Fig. 9A, jasplakinolide/+ plus CA$\mathrm{Abl} /+$ ). We noticed that $\mathrm{F}$-actin staining was weak in neurons treated with jasplakinolide or jasplakinolide plus CA-Abl expression. This was attributable to the competition of jasplakinolide with rhodamine phalloidin for F-actin binding.

When the dendritic number and length were quantified, it became apparent that CA-Abl expression increased both primary dendritic number and length, whereas destabilization of F-actin by latrunculin A promoted primary dendrite number but inhibited dendrite extension (Fig. 9B,C). Treatment of neurons with jasplakinolide showed no significant change in dendritic length but did show a reduction in dendritic branching. These data suggest that F-actin destabilization facilitates dendritic formation and branching. In addition, CA-Abl expression under the background of latrunculin A treatment did not show any additional effects on dendritic branching and only slightly reversed the effects of latrunculin $\mathrm{A}$ on dendrite length, suggesting that latrunculin A effects may be more dominant than CAAbl expression (Fig. 9B,C). Interestingly, CA-Abl expression under the background of jasplakinolide reversed the inhibitory effects of jasplakinolide on dendritic branching but reduced dendritic length. This result suggests that dendritic elongation was limited during the rapid induction of dendrite outgrowth, which is consistent with the process of dendritic arbor formation in vivo. Together, these studies indicate that Abl may function in a dendritic morphogenesis context by destabilizing the local actin cytoskeleton selectively.

We also addressed a possible role for microtubule stability in the CA-Ablinduced branching phenotype (Fig. 10). When microtubules were depolymerized by vincristine treatment, MAP2 staining withdrew from dendritic processes and became localized to the perinuclear cytoplasm of the neuronal cell bodies (Fig. 9, MAP2 staining in CA-Abl/- plus vincristine and $\mathrm{CA}-\mathrm{Abl} /+$ plus vincristine). However, neither control cell morphology nor the CA-Abl phenotypes were reversed, indicating that stabilized microtubules are not required for the maintenance of either morphology. These results are consistent with the hypothesis that the CA-Abl phenotype is dependent on the stability of the actin cytoskeleton but is independent of microtubule stability.

\section{Discussion}

\section{Regulation of dendrogenesis by Abl family kinases}

During development numerous signaling pathways interact to contribute to spatial and temporal regulation of morphogenesis, leading to the correct organization of the brain and the generation of synaptic circuits. Abl family tyrosine kinases contribute to several vital steps during this process, including neurulation and axonogenesis. In this study we attempted to elucidate the contribution of Abl kinases to dendrogenesis, a role for which has not been addressed fully. Treatment with STI571, a small molecule inhibitor of Abl family kinases, altered the developmental dynamics of dendrogenesis in primary rat hippocampal cultures and resulted in a significant simplification of dendritic structure. This outcome ap- 
pears to be independent of axonogenesis, because STI571 treatment occurring after axonal establishment did not have significant effects on continuing axon growth.

We recognize that STI571 also can inhibit PDGF receptor tyrosine kinase in vitro and in fibroblast cells (Carroll et al., 1997; Buchdunger et al., 2000; Simakajornboon et al., 2001). However, AG1296, a selective inhibitor for PDGF receptor, did not affect dendrogenesis. In addition, overexpression of a constitutively active Abl kinase resulted in the outgrowth of an abundant number of highly branched MAP2-positive processes. From these data we conclude that the Abl family members do play a role in dendrogenesis in which kinase activity positively regulates dendrite outgrowth and branching.

The mammalian Abl family of nonreceptor tyrosine kinases is composed of $\mathrm{c}-\mathrm{Abl}$ and Arg, which are encoded by the abl1 and $a b l 2$ genes, respectively. Targeted disruption of $a b l 1$ and $a b l 2$ does not yield discernible phenotypic changes in the context of neuronal morphology (Schwartzberg et al., 1991; Tybulewicz et al., 1991; Koleske et al., 1998). In hippocampal cultures the expression of an Abl kinase-inactive K(290)R mutant (Yuan et al., 1997) showed a moderate trend of reducing dendrite number and length that was statistically insignificant (S. B. Jones and Q. Lu, unpublished results). Similarly, the expression of kinase-inactive Arg did not have significant effects on the morphology of neuronal N2A cells (Hernandez et al., 2004). This indicates that the suppression of both $\mathrm{Abl}$ and Arg kinase activities is required to show a significant inhibitory effect on dendrogenesis. Unfortunately, abl/arg double knock-out mice die before embryonic day 11 , precluding any application of extracting neurons to generate hippocampal cultures (Koleske et al., 1998). Therefore, the ability of STI571 to inhibit selectively both Abl and Arg kinases provides an opportunity to investigate their roles in neuronal development. Our results, focused on the development of dendritic structures, are consistent with previous reports that STI571 treatment during earlier developmental time points resulted in shorter, less branched neurites and that an increase in neurite length occurs with Ablactivation (Zukerberg etal., 2000; Woodring et al., 2002).

\section{Regulation of dendritic complexity by} Abl family tyrosine kinases

A balance between dendrite formation and elongation is critical for the neurons to establish their complex arborization during brain development. Although Abl tyrosine kinase activation promotes both of these
A
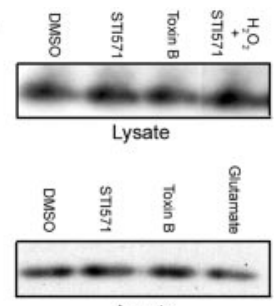

Lysate

Lysate

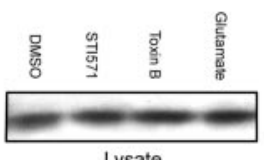

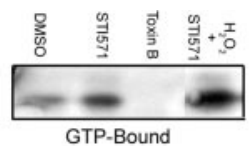

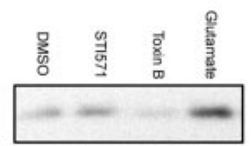

GTP-Bound

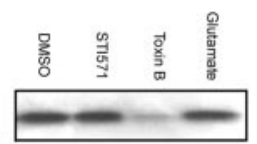

GTP-Bound

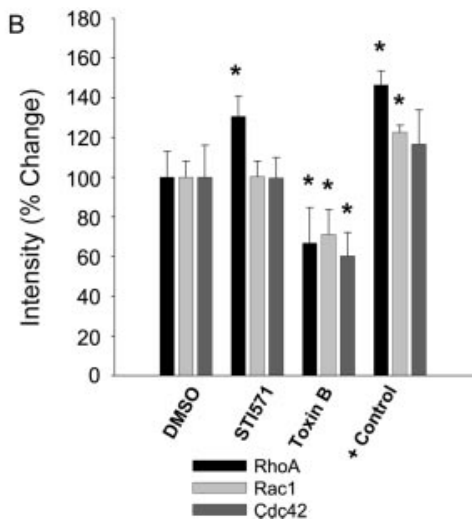

Figure 6. Inhibition of Abl tyrosine kinases by STI571 increases GTP-bound RhoA activity without affecting Rac1 or Cdc42 activity. A, Western blot showing RhoA, Rac1, or Cdc42 in GTP-bound forms. The 5 DIV neurons were treated with DMSO or STI571 for $48 \mathrm{hr}$ or with STI571 for $48 \mathrm{hr}$, followed by ST1571 plus $\mathrm{H}_{2} \mathrm{O}_{2}$ for $10 \mathrm{~min}$. The 6 DIV neurons were treated with Toxin $\mathrm{B}$ for $24 \mathrm{hr}$. The 7 DIV neurons were treated with $100 \mu \mathrm{m}$ glutamate for $10 \mathrm{~min}$. The 7 DIV cell lysates with different treatments were probed with anti-RhoA, Rac1, or Cdc42 antibodies to show their expression levels (left panels). The 7 DIV cell lysates from different treatments also were incubated with GST-RBD (RhoA) or GST-PBD (Rac1 and Cdc42) glutathione beads to pull down GTP-bound RhoA, Rac1, or Cdc42 as an indication of their activities. Bound proteins were analyzed by Western blot with anti-RhoA, Rac1, or Cdc42 antibodies (right panels). B, Relative RhoA, Rac1, and Cdc42 activity was determined semiquantitatively by using densitometric analysis as described in Materials and Methods. Data represent the means \pm SEM of at least three independent experiments; ${ }^{*} p<0.05$
A STI571: Y-27632:
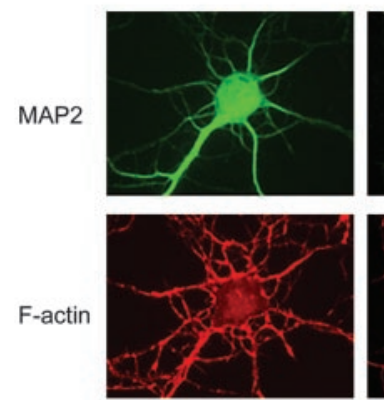

B

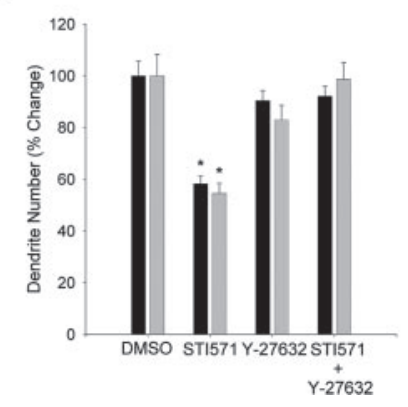

$+$
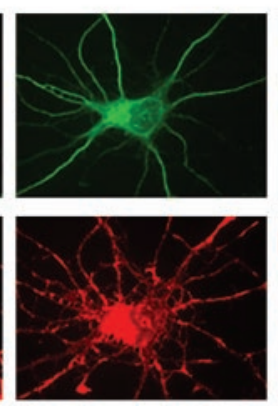

C

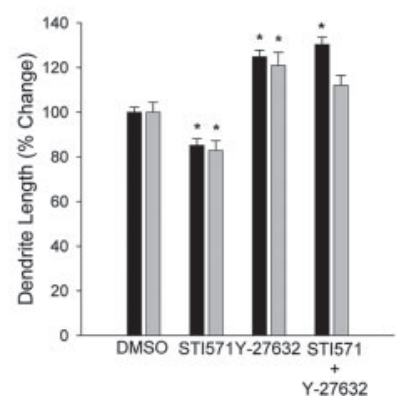

Figure 7. ROCK inhibition suppresses STI571 effect on dendrogenesis. A, Immunofluorescent light microscopy of anti-MAP2 and rhodamine phalloidin staining of 5 DIV hippocampal neurons treated with DMSO (STI571/ - and Y-27632/ -) or $3 \mu \mathrm{m} \mathrm{STI571}$ for $48 \mathrm{hr}$ or treated with $3 \mu \mathrm{m}$ STI571 for $24 \mathrm{hr}$, followed by the addition of Y-27632 for an additional $18 \mathrm{hr}$. Cells were fixed on 7 DIV. Arrowheads point to focal actin filaments. Scale bar, $15 \mu \mathrm{m}$. B, Comparison of the percentage of change of dendrite numbers among neurons treated with DMSO, STI571, Y-27632, and STI571/Y-27632. Note that the reduction of dendrite numbers by STI571 treatment is reversed by coincubation of neurons with Y-27632. C, Comparison of the percentage of change of dendrite length among neurons treated with DMSO, STI571, Y-27632, and STI571/Y-27632. Note that the decrease in dendrite length by STI571 treatment is reversed by coincubation of neurons with $Y-27632$. The effect of $Y-27632$ is more dramatic on the primary dendrites than on the secondary dendrites in neurons 7 DIV. Error bars indicate SEM; ${ }^{*} p<0.05$. 
events, the effects of Abl activation on dendritic formation seem to be more pronounced than on dendrite elongation. This may reflect the fact that the different signaling pathways could regulate dendrite elongation and branching separately. This may not be unique for Abl regulation of dendrogenesis. For example, $\delta$-catenin, a dendrite-specific postsynaptic density protein (Jones et al., 2002), binds to cortactin when dephosphorylated at Src tyrosine kinase sites and promotes an unbranched primary processes. On the other hand, when RhoA is inhibited, $\delta$-catenin enhances the effects of Rho inhibition on branching (Martinez et al., 2003). In our unpublished studies we found that the inhibition of presenilin- $1 / \gamma$-secretase activity induced an exuberant extension of dendrites without significantly affecting dendrite numbers, indicating that the regulation of dendrite formation and elonga- $\begin{array}{lllll}\text { pEGFP-C2: } & + & + & - & - \\ \text { CA-Abl: } & - & + & + & + \\ \text { CA-RhoA-GFP: } & - & - & +\end{array}$
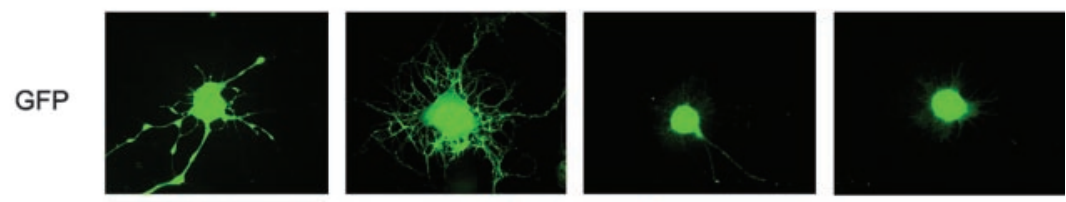

Abl
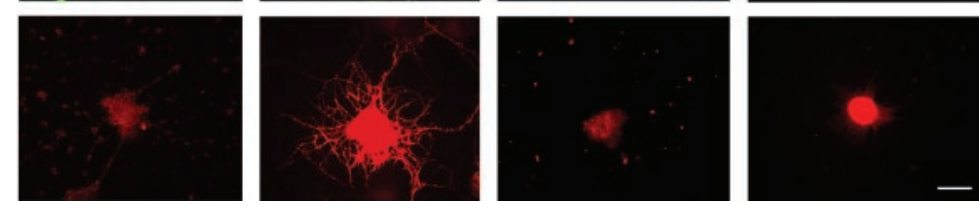

Figure 8. RhoA activation suppresses $C A-A b l$ effect on dendrogenesis. Immunofluorescent light microscopy of hippocampal neurons transfected with pEGFP-C2, pEGFP-C2 plus CA-Abl, pGFP-CA-RhoA, and CA-Abl plus pGFP-CA-RhoA. Top, GFP fluorescence; bottom, anti-Abl. Note that the exuberant dendrite formation in pEGFP-C2 plus CA-Abl-transfected neurons was inhibited by expression of pGFP-CA-RhoA. Note that the Abl immunoreactivity in neurons transfected with pGFP-CA-RhoA is weak because of lower endogenous Abl expression when compared with neurons doubly transfected with pEGFP-C2 plus CA-Abl or CA-Abl plus pGFP-CA-RhoA. Scale bar, $15 \mu \mathrm{m}$.

A

CA-AbI
Latrunculin A
Jasplakinolide

Abl
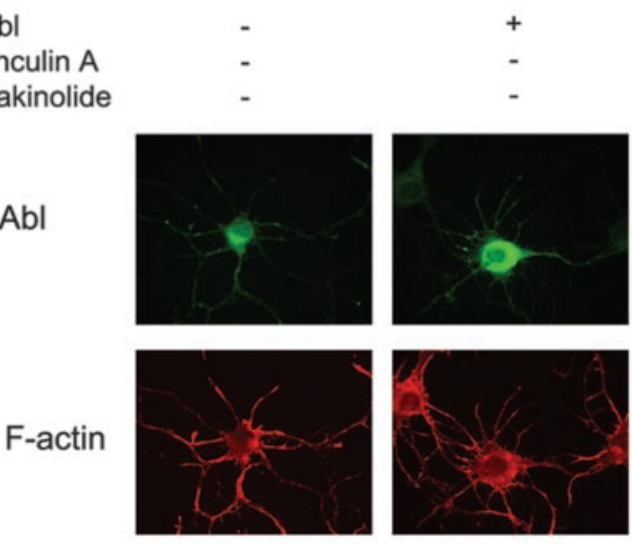

B

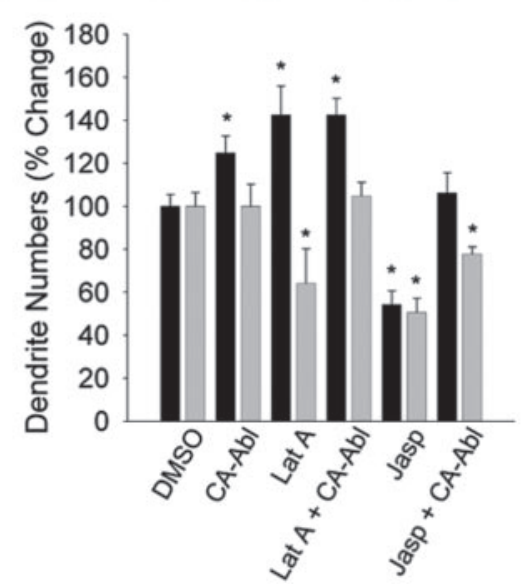

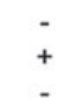
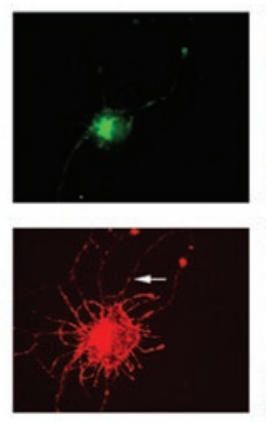

C
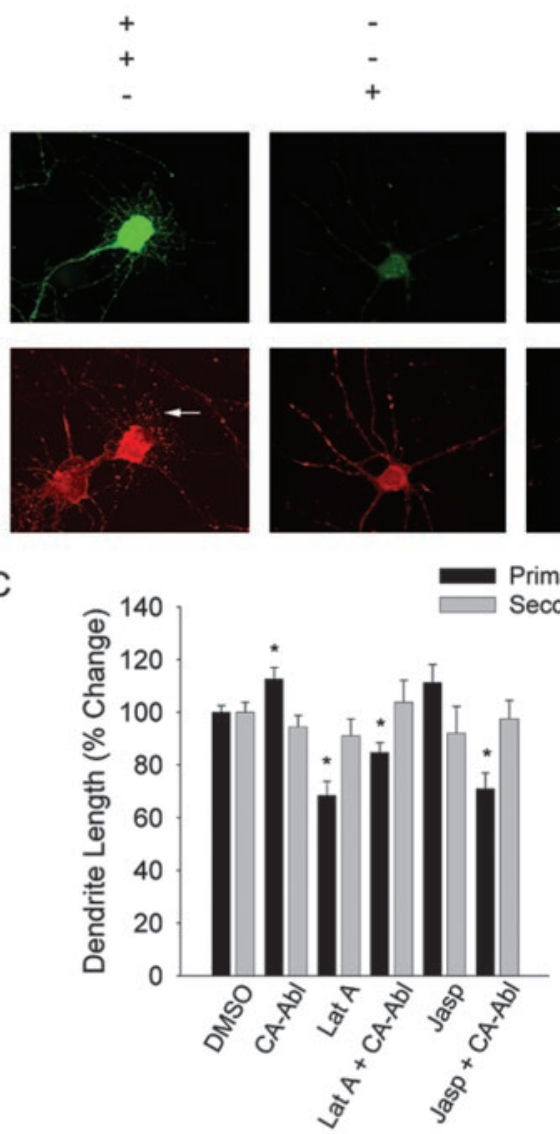

Figure 9. The CA-Abl effect on dendrogenesis is dependent on the reorganization of actin cytoskeleton but is independent of the stability of microtubules. A, Immunofluorescent light microscopy of single neurons by anti-Abl antibody (top) and rhodamine phalloidin (bottom). The 5 DIV hippocampal neurons transfected with CA-Abl were treated either with $5 \mu \mathrm{m}$ latrunculin A the next day for $24 \mathrm{hr}$ to depolymerize actin filament or with $100 \mathrm{~nm}$ jasplakinolide on 7 DIV for $5 \mathrm{hr}$ to stabilize actin filaments. Neurons were fixed on 7 DIV. Note that CA-Abl-induced process outgrowth is suppressed by jasplakinolide. In jasplakinolide-treated neurons, $\mathrm{F}$-actin staining is weak because of competitive binding of jasplakinolide and phalloidin to $\mathrm{F}$-actin. Note that $\mathrm{F}$-actin staining in latrunculin A-treated neurons was enhanced to quantify the processes. Scale bar, $15 \mu \mathrm{m}$. B, Quantification of primary and secondary dendrite outgrowth in neurons incubated with DMSO, latrunculin A, and jasplakinolide as well as transfected with CA-Abl in the presence or absence of latrunculin A and jasplakinolide. C, Quantification of the relative length of primary and secondary dendrites in neurons incubated with DMSO, latrunculin A, and jasplakinolide as well as transfected with CA-Abl in the presence and absence of latrunculin A and jasplakinolide. Dendrite number and length were determined within $120 \mu \mathrm{m}^{2}$ of the single neuronal cell bodies. Data represent the means \pm SEM of three independent experiments; ${ }^{*} p<0.05$. 
tion may be separable. These results appear to echo a dendritic growth model proposed previously in which a single stochastic growth algorithm indicated that, when dendrites actively are forming branches, there is a temporary suppression of additional branching during dendritic arbor formation (Nowakowski et al., 1992).

\section{Regulation of RhoA/ROCK by Abl family kinases}

Our findings led us to question the mechanism by which Abl activity may impact dendrogenesis. Abl kinases are known to organize actin rearrangement both directly, via binding domains for G-actin and F-actin, and indirectly, via interactions with downstream signaling partners (Lanier and Gertler, 2000; Woodring et al., 2003). One group of proteins that seems to be particularly important in many aspects of neuronal development, including dendrogenesis, is the Rho family of small GTPases. In general, RhoA is known to impact neurite outgrowth negatively, whereas Rac1 and Cdc42 increase neurite outgrowth and complexity. Interestingly, we note that inhibition of Abl kinase activity leads to an increase in the activity of RhoA but has no effect on Rac1 or Cdc42 activation. In addition to an increase in RhoA activity, STI571-induced dendritic simplification can be reversed by inhibition of ROCK, a primary downstream effector of RhoA in neurons (Hirose et al., 1998; Bito et al., 2000; Nakayama et al., 2000). Conversely, coexpression of CA-RhoA and CA-Abl is sufficient to suppress CA-Ablgenerated increases in dendrite complexity. These data are consistent with the hypothesis that Abl positively regulates dendrogenesis by inactivating RhoA, which leads to a commensurate decrease in ROCK activity and subsequent increases in dendrite outgrowth and branching.

Regulation of the actin cytoskeleton during dendrogenesis by Abl family kinases

The Abl family kinases are involved in the reorganization of the actin cytoskeleton in a variety of cellular processes. Their roles in neurulation, axon outgrowth, growth cone motility, and neurite branching make them crucial to normal brain development. Here we present evidence that Abl kinase activity is additionally important in cytoskeletal events that underlie dendrogenesis. We found that actin depolymerization with latrunculin A is sufficient to induce the outgrowth of numerous neuritic processes, which are reminiscent of increased dendritic complexity generated by overexpression of CA-Abl kinase. Interestingly, when CA-Ablexpressing cells are subjected to latrunculin A treatment, no additional elaboration of dendritic arbors occurs. Conversely, actin stabilization with jasplakinolide is able to reverse partially the CA-Abl-induced processes. This suggests that Abl activity leads to actin destabilization, which induces dendrite formation. The actin depolymerization has been demonstrated as an important step in promoting neurite extension (Bradke and Dotti, 1999), especially in the context of regulation by proteins of the actindepolymerizing factor (ADF)/cofilin family (Meberg and Bamburg, 2000).

It is interesting to note that, although CA-Abl expression promotes both dendritic extension and branching, latrunculin A treatment promotes dendrite formation while inhibiting den- drite length. Conversely, jasplakinolide treatment has no significant effect on dendrite length but significantly reduces dendritic numbers. In addition, CA-Abl expression was able to reverse the effects of jasplakinolide on dendritic number and, in doing so, limited dendritic elongation. This may be attributable to the inability of actin polymerization to accommodate a rapid increase in dendrite number concurrent with significant elongation. The cellular mechanism regulating the control of dendrite number and length is complex and will require additional investigation. Therefore, it would be important to caution that a sustained destabilization of actin polymerization should limit growth cone activity and suppress neurite extension. Indeed, it is conceivable that actin polymerization and depolymerization must be regulated tightly and locally so that a coordinated formation and extension of neurites can lead to an ultimate establishment of physiologically sound dendritic arbors in vivo. Ample literature exists that demonstrates a variety of extracellular cues in directing the extension and retraction of growth cones and neurites (Liu and Strittmatter, 2001; Gallo and Letourneau, 2004). In addition, intracellular signaling molecules such as Rho family small GTPases regulate local actin cytoskeletal organizations (Kuhn et al., 2000; Luo, 2000). Taken together, our data are consistent with recent studies that demonstrate the importance of RhoA/ROCKdependent actin destabilization in neurite sprouting and axon formation (Bradke and Dotti, 1999; Bito et al., 2000; Da Silva et al., 2003). Thus we propose that Abl family kinases regulate dendrogenesis in developing hippocampal cultures by effecting actin reorganization via a RhoA/ROCK-dependent pathway.

\section{References}

Benard V, Bohl BP, Bokoch GM (1999) Characterization of Rac and Cdc42 activation in chemoattractant-stimulated human neutrophils using a novel assay for active GTPases. J Biol Chem 274:13198-13204.

Bito H, Furuyashiki T, Ishihara H, Shibasaki Y, Ohashi K, Mizuno K, Maekawa M, Ishizaki T, Narumiya S (2000) A critical role for a Rhoassociated kinase, p160ROCK, in determining axon outgrowth in mammalian CNS neurons. Neuron 26:431-441.

Bradke F, Dotti CG (1999) The role of local actin instability in axon formation. Science 283:1931-1934.

Buchdunger E, Zimmermann J, Mett H, Meyer T, Muller M, Druker BJ, Lydon NB (1996) Inhibition of the Abl protein-tyrosine kinase in vitro and in vivo by a 2-phenylaminopyrimidine derivative. Cancer Res 56:100-104.

Buchdunger E, Cioffi CL, Law N, Stover D, Ohno-Jones S, Druker BJ, Lydon NB (2000) Abl protein-tyrosine kinase inhibitor STI571 inhibits in vitro 
signal transduction mediated by c-Kit and platelet-derived growth factor receptors. J Pharmacol Exp Ther 295:139-145.

Carroll M, Ohno-Jones S, Tamura S, Buchdunger E, Zimmermann J, Lydon NB, Gilliland DG, Druker BJ (1997) CGP 57148, a tyrosine kinase inhibitor, inhibits the growth of cells expressing BCR-ABL, TEL-ABL, and TEL-PDGFR fusion proteins. Blood 90:4947-4952.

Da Silva JS, Medina M, Zuliani C, Di Nardo A, Witke W, Dotti CG (2003) RhoA/ROCK regulation of neuritogenesis via profilin IIa-mediated control of actin stability. J Cell Biol 162:1267-1279.

Dotti CG, Sullivan CA, Banker GA (1988) The establishment of polarity by hippocampal neurons in culture. J Neurosci 8:1454-1468.

Finn AJ, Feng G, Pendergast AM (2003) Postsynaptic requirement for Abl kinases in assembly of the neuromuscular junction. Nat Neurosci 6:717-723.

Fischer M, Kaech S, Wagner U, Brinkhaus H, Matus A (2000) Glutamate receptors regulate actin-based plasticity in dendritic spines. Nat Neurosci 3:887-894.

Gallo G, Letourneau PC (2004) Regulation of growth cone actin filaments by guidance cues. J Neurobiol 58:92-102.

Goslin K, Asmussen H, Banker G (1998) Rat hippocampal neurons in low density culture. In: Culturing nerve cells (Goslin K, ed), pp 339-370. Cambridge, MA: MIT.

Hernandez SE, Settleman J, Koleske AJ (2004) Adhesion-dependent regulation of p190RhoGAP in the developing brain by the Abl-related gene tyrosine kinase. Curr Biol 14:691-696.

Hirose M, Ishizaki T, Watanabe N, Uehata M, Kranenburg O, Moolenaar WH, Matsumura F, Maekawa M, Bito H, Narumiya S (1998) Molecular dissection of the Rho-associated protein kinase (p160ROCK)-regulated neurite remodeling in neuroblastoma N1E-115 cells. J Cell Biol 141:1625-1636.

Jones SB, Lanford GW, Chen YH, Moribito M, Kim K, Lu Q (2002) Glutamate-induced $\delta$-catenin redistribution and dissociation from postsynaptic receptor complexes. Neuroscience 115:1009-1021.

Katoh H, Aoki J, Ichikawa A, Negishi M (1998) p160 RhoA-binding kinase ROK $\alpha$ induces neurite retraction. J Biol Chem 273:2489-2492.

Kim K, Sirota A, Chen YH, Jones SB, Dudek R, Lanford GW, Thakore C, Lu Q (2002) Dendrite-like process formation and cytoskeletal remodeling regulated by $\delta$-catenin expression. Exp Cell Res 275:171-184.

Koleske AJ, Gifford AM, Scott ML, Nee M, Bronson RT, Miczek KA, Baltimore D (1998) Essential roles for the Abl and Arg tyrosine kinases in neurulation. Neuron 21:1259-1272.

Kranenburg O, Poland M, van Horck FP, Drechsel D, Hall A, Moolenaar WH (1999) Activation of RhoA by lysophosphatidic acid and $\mathrm{G} \alpha_{12 / 13}$ subunits in neuronal cells: induction of neurite retraction. Mol Biol Cell 10:1851-1857.

Kuhn TB, Meberg PJ, Brown MD, Bernstein BW, Minamide LS, Jensen JR, Okada K, Soda EA, Bamburg JR (2000) Regulating actin dynamics in neuronal growth cones by ADF/cofilin and Rho family GTPases. J Neurobiol 44:126-144.

Lanier LM, Gertler FB (2000) From Abl to actin: Abl tyrosine kinase and associated proteins in growth cone motility. Curr Opin Neurobiol 10:80-87.

Lee T, Winter C, Marticke SS, Lee A, Luo L (2000) Essential roles of Drosophila RhoA in the regulation of neuroblast proliferation and dendritic but not axonal morphogenesis. Neuron 25:307-316.

Leeuwen FN, Kain HE, Kammen RA, Michiels F, Kranenburg OW, Collard JG (1997) The guanine nucleotide exchange factor Tiam1 affects neuronal morphology; opposing roles for the small GTPases Rac and Rho. J Cell Biol 139:797-807.

Li JH, Wang YH, Wolfe BB, Krueger KE, Corsi L, Stocca G, Vicini S (1998) Developmental changes in localization of NMDA receptor subunits in primary cultures of cortical neurons. Eur J Neurosci 10:1704-1715.

Liebl EC, Forsthoefel DJ, Franco LS, Sample SH, Hess JE, Cowger JA, Chandler MP, Shupert AM, Seeger MA (2000) Dosage-sensitive, reciprocal genetic interactions between the Abl tyrosine kinase and the putative GEF trio reveal trio's role in axon pathfinding. Neuron 26:107-118.

Liu BP, Strittmatter SM (2001) Semaphorin-mediated axonal guidance via Rho-related G-proteins. Curr Opin Cell Biol 13:619-626.

Lu Q, Mukhopadhyay NK, Griffin JD, Paredes M, Medina M, Kosik KS
(2002) Brain armadillo protein $\delta$-catenin interacts with Abl tyrosine kinase and modulates cellular morphogenesis in response to growth factors. J Neurosci Res 67:618-624.

Luo L (2000) Rho GTPases in neuronal morphogenesis. Nat Rev Neurosci 1:173-180.

Martinez MC, Ochiishi T, Majewski M, Kosik KS (2003) Dual regulation of neuronal morphogenesis by a $\delta$-catenin-cortactin complex and Rho. J Cell Biol 162:99-111.

McAllister AK (2000) Cellular and molecular mechanisms of dendrite growth. Cereb Cortex 10:963-973.

Meberg PJ, Bamburg JR (2000) Increase in neurite outgrowth mediated by overexpression of actin depolymerizing factor. J Neurosci 20:2459-2469.

Moresco EM, Koleske AJ (2003) Regulation of neuronal morphogenesis and synaptic function by Abl family kinases. Curr Opin Neurobiol 13:535-544.

Moresco EM, Scheetz AJ, Bornmann WG, Koleske AJ, Fitzsimonds RM (2003) Abl family nonreceptor tyrosine kinases modulate short-term synaptic plasticity. J Neurophysiol 89:1678-1687.

Nakayama AY, Harms MB, Luo L (2000) Small GTPases Rac and Rho in the maintenance of dendritic spines and branches in hippocampal pyramidal neurons. J Neurosci 20:5329-5338.

Nowakowski RS, Hayes NL, Egger MD (1992) Competitive interactions during dendritic growth: a simple stochastic growth algorithm. Brain Res 576:152-156.

Pendergast AM (2002) The Abl family kinases: mechanisms of regulation and signaling. Adv Cancer Res 85:51-100.

Portera-Cailliau C, Pan DT, Yuste R (2003) Activity-regulated dynamic behavior of early dendritic protrusions: evidence for different types of dendritic filopodia. J Neurosci 23:7129-7142.

Rao A, Craig AM (2000) Signaling between the actin cytoskeleton and the postsynaptic density of dendritic spines. Hippocampus 10:527-541.

Ren XD, Kiosses WB, Schwartz MA (1999) Regulation of the small GTPbinding protein Rho by cell adhesion and the cytoskeleton. EMBO J 18:578-585.

Schwartzberg PL, Stall AM, Hardin JD, Bowdish KS, Humaran T, Boast S, Harbison ML, Robertson EJ, Goff SP (1991) Mice homozygous for the ablm1 mutation show poor viability and depletion of selected $\mathrm{B}$ and $\mathrm{T}$ cell populations. Cell 65:1165-1175.

Simakajornboon N, Szerlip NJ, Gozal E, Anonetapipat JW, Gozal D (2001) In vivo PDGF $\beta$ receptor activation in the dorsocaudal brainstem of the rat prevents hypoxia-induced apoptosis via activation of Akt and BAD. Brain Res 895:111-118.

Sin WC, Haas K, Ruthazer ES, Cline HT (2002) Dendrite growth increased by visual activity requires NMDA receptor and Rho GTPases. Nature 419:475-480.

Tybulewicz VL, Crawford CE, Jackson PK, Bronson RT, Mulligan RC (1991) Neonatal lethality and lymphopenia in mice with a homozygous disruption of the c-Abl proto-oncogene. Cell 65:1153-1163.

Uehata M, Ishizaki T, Satoh H, Ono T, Kawahara T, Morishita T, Tamakawa H, Yamagami K, Inui J, Maekawa M, Narumiya S (1997) Calcium sensitization of smooth muscle mediated by a Rho-associated protein kinase in hypertension. Nature 389:990-994.

Van Etten RA (1999) Cycling, stressed-out and nervous: cellular functions of c-Abl. Trends Cell Biol 9:179-186.

West AE, Neve RL, Buckley KM (1997) Identification of a somatodendritic targeting signal in the cytoplasmic domain of the transferrin receptor. J Neurosci 17:6038-6047.

Woodring PJ, Litwack ED, O'Leary DD, Lucero GR, Wang JY, Hunter T (2002) Modulation of the F-actin cytoskeleton by c-Abl tyrosine kinase in cell spreading and neurite extension. J Cell Biol 156:879-892.

Woodring PJ, Hunter T, Wang JY (2003) Regulation of F-actin-dependent processes by the Abl family of tyrosine kinases. J Cell Sci 116:2613-2626.

Yuan ZM, Huang Y, Ishiko T, Kharbanda S, Weichselbaum R, Kufe D (1997) Regulation of DNA damage-induced apoptosis by the c-Abl tyrosine kinase. Proc Natl Acad Sci USA 94:1437-1440.

Zukerberg LR, Patrick GN, Nikolic M, Humbert S, Wu CL, Lanier LM, Gertler FB, Vidal M, Van Etten RA, Tsai LH (2000) Cables links Cdk5 and $\mathrm{c}-\mathrm{Abl}$ and facilitates $\mathrm{Cdk} 5$ tyrosine phosphorylation, kinase upregulation, and neurite outgrowth. Neuron 26:633-646. 